\title{
Stochastic bifurcation characteristics of SMA intravascular stent subjected to radial and axial excitations
}

\author{
Zhiwen Zhu ${ }^{\text {a }}$, Wendi Zhang a and Jia Xu, ${ }^{\text {a, }, *}$ \\ ${ }^{a}$ Deparment of Mechanics, Tianjin University, 92 Weijin Road, Nankai District, Tianjin 300072, \\ China \\ ${ }^{\mathrm{b}}$ Tianjin Key Laboratory of Nonlinear Dynamics and Chaos Control, 92 Weijin Road, Nankai District, \\ Tianjin 300072, China
}

\begin{abstract}
A kind of shape memory alloy (SMA) hysteretic nonlinear model is developed, and the stochastic bifurcation characteristics of SMA intravascular stents subjected to radial and axial excitations are studied in this paper. A new nonlinear differential item is introduced to interpret the hysteretic phenomena of SMA strain-stress curves, and the dynamic model of SMA intravascular stent subjected to radial and axial stochastic excitations is established. The conditions of the system's stochastic stability are determined, and the probability density function of the system response is obtained. Finally, the stochastic Hopf bifurcation characteristics of the system are analyzed. Theoretical analysis and numerical simulation show that the system stability varies with bifurcation parameters, and stochastic Hopf bifurcation occurs in the process; there are two limit cycles in the stationary probability density of the system response in some cases, which means that there are two vibration amplitudes whose probability are both very high; jumping phenomena between the two vibration amplitudes appears with the change of conditions, which may cause stent fracture or loss. The results of this paper are helpful for application of SMA intravascular stent in biomedical engineering fields.
\end{abstract}

Keywords: Shape Memory Alloy (SMA), intravascular stent, stochastic load, global stability, stochastic bifurcation

\section{Introduction}

Shape Memory Alloy (SMA) is an ideal material for intravascular stents due to its shape memory characteristics, corrosion resistance and biocompatibility. SMA intravascular stents have been applied in clinical treatment to intravascular lesion stenosis widely. It is fixed in artery by friction in the course of surgery. Subjected to the radial pressure from the vascular wall and the axial viscous force from blood, the SMA intravascular stent has complex response, which causes its vibration and loss. For avoiding stent loss, it is necessary to study the dynamic characteristics of SMA intravascular stent subjected to the radial pressure from vascular wall and the axial viscous force from blood.

\footnotetext{
${ }^{*}$ Corresponding author: Jia Xu, Department of Mechanics, Tianjin University, 92 Weijin Road, Nankai District, Tianjin 300072, China. Tel.: +8627401981; Fax:+8627401981; E-mail: xujia@tju.edu.cn.
}

0959-2989/14/\$27.50 @ 2014 - IOS Press and the authors. 
Many scholars studied the applications of SMA stent in biomedical engineering [1-8]. Longas proposed a NiTi stent and test its mechanical properties firstly [1]. Kaoriuri et al. applied TiNi shape memory alloy foil in self-deployable origami stent grafts [2]. Verschuur et al. designed a new esophageal Niti-S stent for the prevention of migration [3]. Puertolas et al. studied the behavior of a bellshaped colonic self-expandable NiTi stent under peristaltic movements [4]. Auricchio et al. established the model of SMA stent and analyzed its deployment in finite element method [5]. Zhu et al. developed a SMA nonlinear constructive model [6]. Although many achievements were obtained in the past years, most of them were focused on design and deformation analysis, and the theoretical results of the dynamic characteristics of SMA stent were not abundant. To patients who have arrhythmia, the radial pressure from the vascular wall is irregular and can be assumed as a harmonic wave added a stochastic excitation, while the axial viscous force from blood changes slowly due to the fluid continuity of the blood. In this paper, the stochastic bifurcation characteristics of SMA intravascular stent subjected to radial and axial excitations were studied.

For analyzing the dynamic characteristics of SMA intravascular stent, the accurate constitutive model of SMA is needed. However, it is hard to establish an accurate model of the SMA due to its complex nonlinear hysteretic characteristics. Most of the current SMA models are shown as equations with subsection functions or double integral functions, which cause the difficulty to analyze the dynamic characteristics of SMA stent in theory $[7,8]$. In this paper, a new nonlinear differential model is introduced to interpret the hysteretic phenomena of SMA strain-stress curves, the nonlinear dynamic model of SMA intravascular stent subjected to radial and axial excitations is developed, and the stochastic bifurcation characteristics of the system are analyzed.

\section{Material and methods}

The strain-stress curves of the SMA are presented in Figure 1. In this paper, a new nonlinear differential model is introduced to interpret the hysteretic strain-stress curves of the SMA as follows:

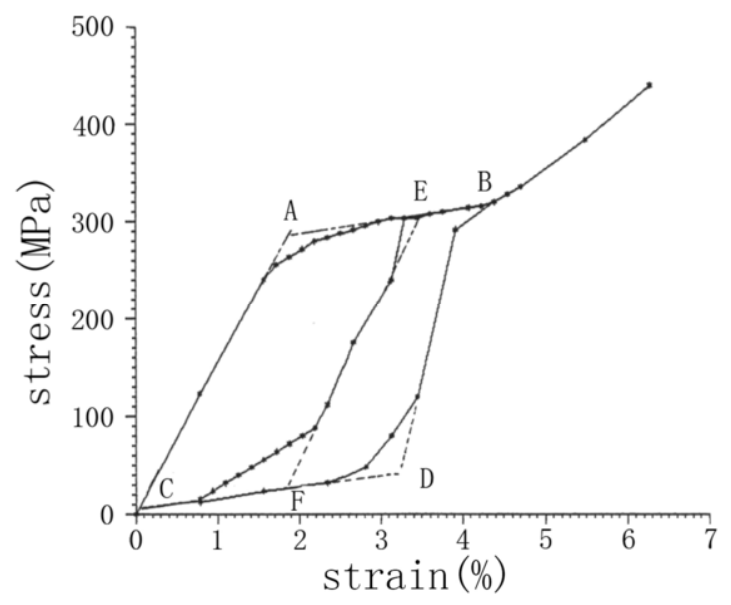

Fig. 1. Strain-stress curves of the SMA.

$$
\sigma=\sigma_{1}+\sigma_{2}=a_{1} \varepsilon+a_{2} \varepsilon^{2}+a_{3} \varepsilon^{3}+\left(a_{4} \varepsilon+a_{5} \varepsilon^{2}+a_{6} \varepsilon^{3}+a_{7} \varepsilon^{4}+a_{8} \varepsilon^{5}+a_{9} \varepsilon^{6}+a_{10} \varepsilon^{7}+a_{11} \varepsilon^{8}\right) \dot{\varepsilon}
$$


where $\sigma$ is the stress, $\varepsilon$ is the strain, $a_{i}(\mathrm{i}=1 \sim 11)$ are coefficients, $\sigma_{1}=a_{1} \varepsilon+a_{2} \varepsilon^{2}+a_{3} \varepsilon^{3}$ is skeleton curve of the hysteresis loop, $\sigma_{2}=\left(a_{4} \varepsilon+a_{5} \varepsilon^{2}+a_{6} \varepsilon^{3}+a_{7} \varepsilon^{4}+a_{8} \varepsilon^{5}+a_{9} \varepsilon^{6}+a_{10} \varepsilon^{7}+a_{11} \varepsilon^{8}\right) \dot{\varepsilon}$ is the new nonlinear differential item. To SMA shown in Figure 1, $a_{1}=70.5, a_{2}=-0.32, a_{3}=0.057$.

Partial least-square regression method is used to test the fitting effect of Eq. (1). The analyze result of principal component based on experimental data is shown in Figure 2, and the coefficient values are shown in Figure 3, in which VIP is variable importance. In Figure 2, there isn't any item whose VIP is evidently little, which means that each item should be reserved in the constructive model.

The results of DModX and DModY are shown in Figures 4 and 5, in which residual standard deviation (RSD) of samples are shown in X space and Y space. X space is the data space of the independent variable, and $\mathrm{Y}$ space is the data space of the dependent variable. Only some points are singular points, which means that most of the forecasted data are effective.

The result of forecast test to Eq. (1) is shown in Figure 6, where the red line presents the real data and the black line presents the forecast value. Eq. (1) can describe the real curve well.

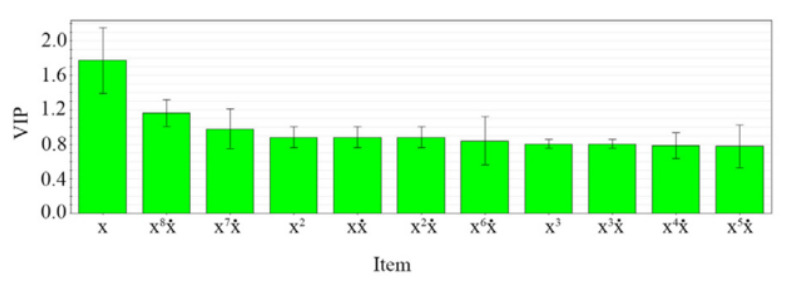

Fig. 2. Variable importance of each item.

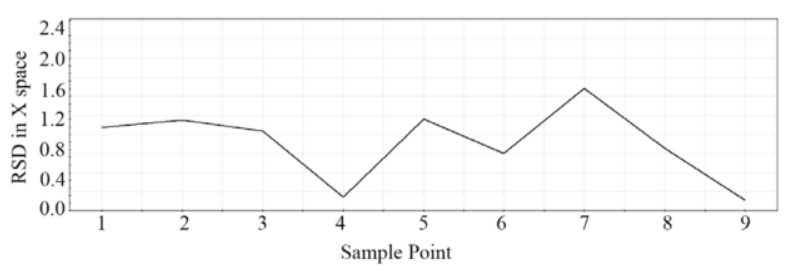

Fig. 4. RSD in X space.

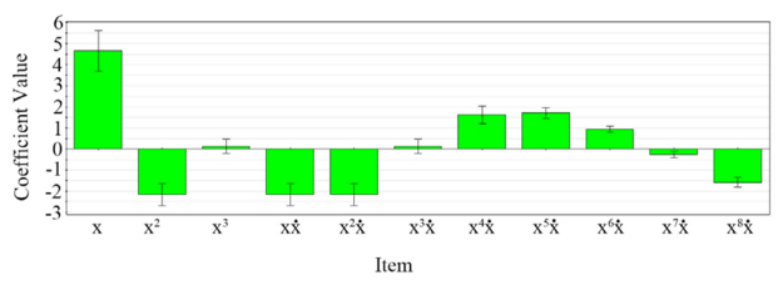

Fig. 3. Coefficient values of each item.

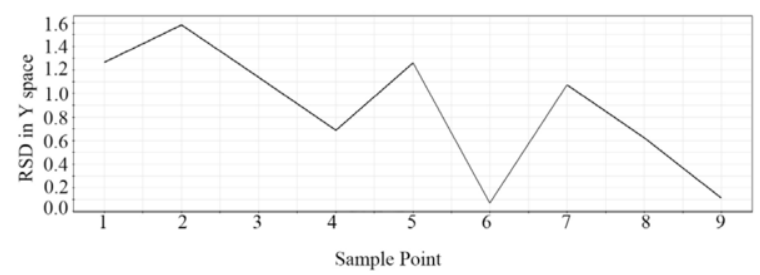

Fig. 5. RSD in Y space.

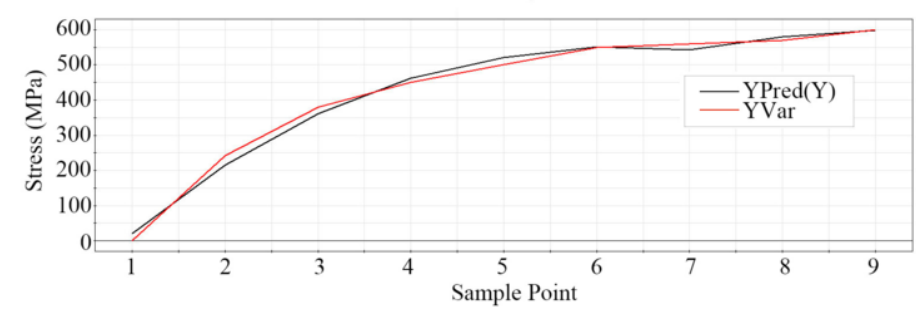

Fig. 6. Results of forecast test for the fitting effect of Eq. (1) on strain-stress data of SMA. 


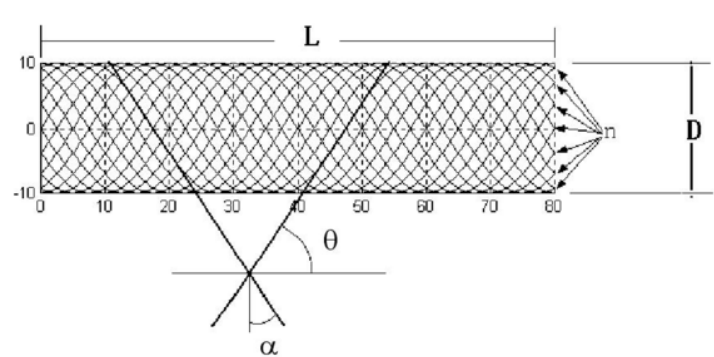

Fig. 7. Structure of SMA intravascular stent.

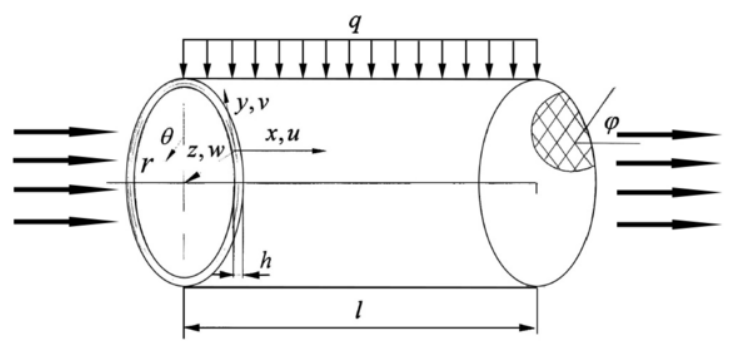

Fig. 8. Mechanical model of SMA intravascular stent.

\section{Nonlinear dynamic model and stochastic stability analysis of SMA intravascular stent subjected to radial and axial excitations}

The structure of SMA intravascular stent is shown in Figure 7. The structure can be simplified as thin cylindrical shell shown in Figure 8.

The dynamic model of SMA intravascular stent under radial loads can be shown as follows:

$$
\rho h r^{2} \frac{\partial^{2} w}{\partial t^{2}}=q r-\frac{\partial^{2} M_{\theta}}{r \partial \theta^{2}}-N_{\theta}\left(1-\frac{\partial^{2} w}{r \partial \theta^{2}}+F_{v}\right)
$$

where $\rho$ is the density of SMA, $h$ is the thickness of SMA stent, $r$ is the radius of SMA stent, $w$ is vibration mode, $w=w(t, \theta)=u(t) \sin k \theta, \theta$ is the torsion angle, $N_{\theta}$ is the shearing stress, $M_{\theta}$ is the torque, $q$ is the radial pressure from the vascular wall, $F_{v}$ is the axial viscous force from the blood. To patients who have arrhythmia, the radial pressure from the vascular wall is irregular and can be assumed as a harmonic wave added to a stochastic excitation, while the axial viscous force from the blood changes slowly due to the fluid continuity of the blood. Thus, $q=a \cos \Omega t+b \varsigma_{1}(t)$ and $F_{v}=c \cos \Omega t+d \varsigma_{2}(t), b$ and $d$ are the intensities of radial and axial stochastic excitation, $a$ and $c$ are the intensities of harmonic wave, $\varsigma_{1}(t)$ and $\varsigma_{2}(t)$ is standard Gauss white noises, their means are zero and their intensities are $2 \mathrm{D}$.

Let $w(t, \theta)=u(t) \sin k \theta$, where $u(t)$ is the radial vibration amplitude. Thus, the dynamic equation of radial vibration amplitude of SMA stent can be solved from Eq. (2) as follows:

$$
\ddot{u}+\left(\eta_{1}+\eta_{2} u^{2}+\eta_{3} u^{4}+\eta_{4} u^{6}+\eta_{5} u^{8}\right) \dot{u}+\eta_{6} u+\eta_{7} u^{3}=e_{1} u \varsigma_{1}(\mathrm{t})+e_{2} \cos \Omega t+e_{3} \varsigma_{2}(t)
$$


where $\eta_{1}=\frac{c}{\rho h r^{2}}, \eta_{2}=\frac{3 a_{5} I \pi^{8}}{4 \rho h r^{2}}, \eta_{3}=\frac{5 a_{7} I \pi^{12}}{8 \rho h r^{2}}, \eta_{4}=\frac{7 a_{9} I \pi^{16}}{16 \rho h r^{2}}, \eta_{5}=\frac{9 a_{11} I \pi^{24}}{64 \rho h r^{2}}, \eta_{6}=\frac{a_{1} I \pi^{4}}{\rho h r^{2}}$, $\eta_{7}=\frac{3 a_{3} I \pi^{8}}{4 \rho h r^{2}}, e_{1}=\frac{b \pi^{2}}{\rho h r^{2}}, e_{2}=\frac{(3 a+c) \pi^{2}}{4 \rho h r^{2}}, e_{3}=\frac{d \pi^{2}}{\rho h r^{2}}$.

Let $u=q, \dot{u}=p$, then Eq. (3) can also be rewritten as follows:

$$
\left\{\begin{array}{l}
\dot{q}=p \\
\dot{p}=-\eta_{6} q-\eta_{7} q^{3}-\left(\eta_{1}+\eta_{2} q^{2}+\eta_{3} q^{4}+\eta_{4} u^{6}+\eta_{5} u^{8}\right) p+e_{1} u \varsigma_{1}(\mathrm{t})+e_{2} \cos \Omega t+e_{3} \varsigma_{2}(t)
\end{array}\right.
$$

Let the system's Hamiltonian function be $H=\frac{1}{2} p^{2}+\frac{1}{2} \eta_{6} q^{2}$, then the averaged Ito equation about Eq. (4) can be shown as follows:

$$
d H=m(H) d t+\sigma(H) d B(t)
$$

where $B(t)$ is standard Wiener process, $m(H)$ and $\sigma(H)$ are drift and diffusion coefficients,

$$
\begin{aligned}
& m(H)=D e_{3}^{2}+\left(\frac{D e_{1}^{2}}{\eta_{6}}-\eta_{1}\right) H-\frac{\eta_{2}}{2 \eta_{6}} H^{2}-\frac{\eta_{3}}{2 \eta_{6}^{2}} H^{3}-\frac{5 \eta_{4}}{8 \eta_{6}^{3}} H^{4}-\frac{7 \eta_{5}}{8 \eta_{6}^{4}} H^{5} \\
& \sigma^{2}(H)=2 D e_{3}^{2} H+\frac{D e_{1}^{2} H^{2}}{\eta_{6}}
\end{aligned}
$$

When

$$
\begin{aligned}
& H \rightarrow 0: \quad m(H) \rightarrow \mathrm{O}\left(H^{0}\right), \sigma^{2}(H) \rightarrow \mathrm{O}\left(H^{1}\right) \\
& H \rightarrow \infty: m(H) \rightarrow \mathrm{O}\left(H^{5}\right), \sigma^{2}(H) \rightarrow \mathrm{O}\left(H^{2}\right)
\end{aligned}
$$

So

$$
\alpha_{l}=1, \beta_{l}=0, c_{l}=2 ; \alpha_{r}=2, \beta_{r}=5, m(+\infty)=-\frac{7 \eta_{5}}{8 \eta_{6}^{4}} H^{5}
$$

where $\alpha_{l}$ is diffusion exponent, $\beta_{l}$ is drift exponent, $c_{l}$ is character value, $l$ is left boundary, $r$ is the right boundary.

According to the singular boundary theory:

1. the left boundary $H=0$ is entrance boundary

2. the right boundary $H=\infty$ is entrance boundary if $\eta_{5}>0$;

3. the right boundary $H=\infty$ is regular boundary if $\eta_{5}=0$; 
4. the right boundary $H=\infty$ is exit boundary if $\eta_{5}<0$;

The system's globally stability is presented in Figure 9. We can see that the system is globally asymptotic unstable when $\eta_{5}<0$, and the necessary and sufficient conditions for globally asymptotic stability of the trivial solution is $\eta_{5}>0$. Thus, the trivial solution $H=\bar{H}=$ const is globally asymptotically stable only $\eta_{5}>0$, which means that the system's motion is periodic.

\section{Stochastic bifurcation analysis and simulation result}

The averaged FPK equation of Eq. (5) is:

$$
\frac{\partial f}{\partial t}=-\frac{\partial}{\partial H}[m(H) f]+\frac{1}{2} \frac{\partial^{2}\left[\sigma^{2}(H) f\right]}{\partial H^{2}}
$$

where $f$ is the stationary probability density of system response,

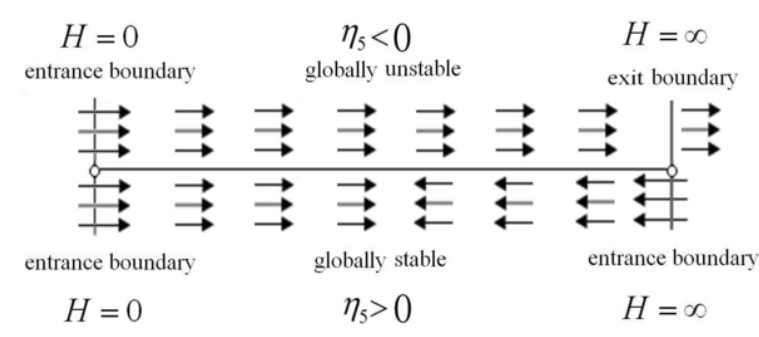

Fig. 9. System's globally stability.

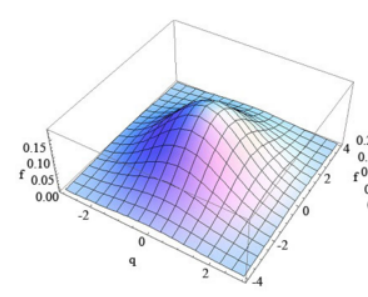

(a)

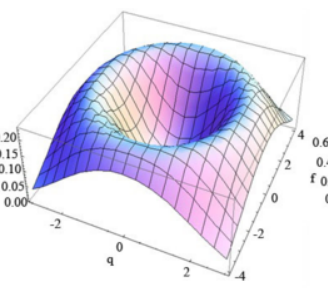

(b)

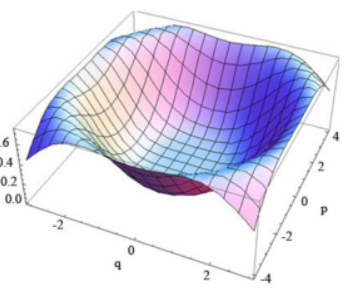

(c)

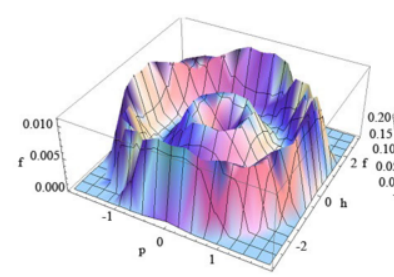

(d)

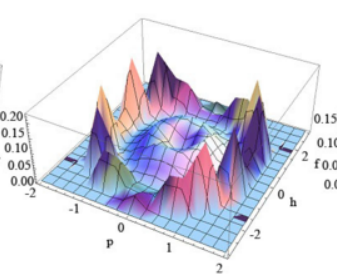

(e)

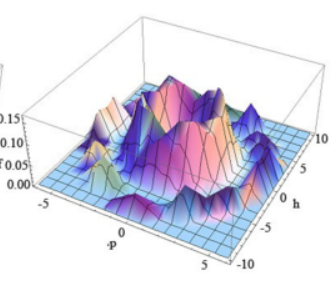

(f)

Fig. 10. Stationary probability density of the system response. 


$$
\begin{aligned}
& f(H)=\bar{A}\left(H+\frac{2 e_{3}^{2} \eta_{6}}{e_{1}^{2}}\right)^{\frac{-2 \eta \eta_{6}}{\rho_{e}}} \exp \left[-\frac{\eta_{2}}{D e_{1}^{2}}\left(H+\frac{2 e_{3}^{2} \eta_{6}}{e_{1}^{2}}\right)-\frac{\eta_{3}}{4 D e_{1}^{2} \eta_{4}^{2}}\left(H+\frac{2 e_{3}^{2} \eta_{6}}{e_{1}^{2}}\right)^{2}\right. \\
& \left.-\frac{5 \eta_{4}}{12 D e_{1}^{2} \eta_{6}^{2}}\left(H+\frac{2 e_{3}^{2} \eta_{6}}{e_{1}^{2}}\right)^{3}-\frac{7 \eta_{5}}{16 D e_{1}^{2} \eta_{6}^{3}}\left(H+\frac{2 e_{3}^{2} \eta_{6}}{e_{1}^{2}}\right)^{4}\right]
\end{aligned}
$$

where $\bar{A}$ is a normalization constant.

The numerical simulation results are presented in Figure 10, where $\eta_{1}=0.1, \eta_{6}=100, D=0.5$, $b_{3}=150, l=1, M=40, E=2 \times 10^{11}, A=8 \times 10^{-4}, I=6 \times 10^{-11}$.

$$
\begin{aligned}
& \text { (a) } \eta_{i}=0(i=2 \sim 5,7) ; \\
& \text { (b) } \eta_{2}=-0.5, \eta_{7}=0.5, \eta_{i}=0(i=3 \sim 5) \\
& \text { (c) } \eta_{2}=-1, \eta_{7}=1, \eta_{i}=0(i=3 \sim 5) \\
& \text { (d) } \eta_{2}=-0.5, \eta_{3}=0.02, \eta_{4}=-0.0015, \eta_{5}=0.00125, \eta_{7}=0.5 \\
& \text { (e) } \eta_{2}=-1, \eta_{3}=0.02, \eta_{4}=-0.001, \eta_{5}=0.00125, \eta_{7}=0.5 \\
& \text { (f) } \eta_{2}=-0.5, \eta_{3}=0.02, \eta_{4}=-0.002, \eta_{5}=0.0015, \eta_{7}=0.5
\end{aligned}
$$

From Figure 10:

1. $\eta_{1}$ is the linear damping coefficient, $\eta \geq 0 ; \eta_{6}$ is the linear stiffness coefficient, $\eta_{6} \geq 0 ; D$ is intensity of Gauss white noise, $D>0$.Thus, the exponent $-\frac{2 \eta_{1} \eta_{6}}{D e^{2}}$ is always negative or zero;

2. In a linear system, the nonlinear damping and stiffness of the substrate are both zero, which means that $\eta_{i}=0(i=2 \sim 5,7)$. The stationary probability density $f(H)$ is determined only by the item $\left(H+\frac{2 e_{3}^{2} \eta_{6}}{e_{1}^{2}}\right)^{-\frac{2 \eta_{1} \eta_{6}}{D e^{2}}}$. The item $\left(H+\frac{2 e_{3}^{2} \eta_{6}}{e_{1}^{2}}\right)^{-\frac{2 \eta_{1} \eta_{6}}{D e^{2}}}$ decreases when $H$ increases since the exponent $-\frac{2 \eta_{1} \eta_{6}}{D e^{2}}$ is always negative, and thus, the stationary probability density of $H=0$ is the maximum, which is shown in Figure 10(a);

3. In a nonlinear system, the stationary probability density $f(H)$ is determined by Eq. (9). When $H$ is significantly little, the item $\left(H+\frac{2 e_{3}^{2} \eta_{6}}{e_{1}^{2}}\right)^{-\frac{2 \eta_{1} \eta_{6}}{D e^{2}}}$ becomes remarkable; when $H$ is sufficiently large, the item $\exp \left[-\frac{7 \eta_{5}}{16 D e_{1}^{2} \eta_{6}^{3}}\left(H+\frac{2 e_{3}^{2} \eta_{6}}{e_{1}^{2}}\right)^{4}\right]$ is remarkable.

4a) when $\eta_{1}>0, \eta_{2}<0, \eta_{7}>0$ and $\eta_{i}=0 \quad(i=3 \sim 5)$, the stationary probability density $f(H)$ initially decreases, increases, and finally decreases when $H$ increases. A limit loop is found in the figures of stationary probability density, which are shown in Figure 10(b) and Figure 10(c); the limit loop increase with the parameters $\eta_{2}$ and $\eta_{7}$. Limit cycle means that selfexcited vibration are induced by the hysteretic nonlinear characteristics of SMA since the parameters $\eta_{2}$ and $\eta_{7}$ are the nonlinear coefficients of SMA; 
4b) when $\eta_{1}>0, \eta_{2}<0, \eta_{3}>0, \eta_{4}<0, \eta_{5}>0$ and $\eta_{7}>0$, Two limit cycles are found in the stationary probability density, which are shown in Figures 10(d), 10(e) and 10(f). Two limit cycles mean that there are two vibration amplitudes whose probability are both sufficiently high, and jumping phenomena between the two vibration amplitudes appear with the change to conditions, which should cause stents loss;

4c) when $\eta_{5}<0$, the stationary probability density $f(H)$ finally increases. However, the stationary probability density of the system cannot be infinite. Thus, the system becomes unstable when $\eta_{5}<0$;

4. In sum, the stationary probability density of the system response can be changed through adjusting the parameters $\eta_{i}$. It means that different SMA materials cause different vibration amplitudes of the system because the parameters $\eta_{i}$ are determined by the substrate material. It provides a way to reduce the vibration of SMA stent.

\section{Conclusion}

A kind of shape memory alloy (SMA) hysteretic nonlinear model is developed, and the stochastic bifurcation characteristics of SMA intravascular stents subjected to radial and axial excitations are studied in this paper. A new differential item is introduced to interpret the hysteretic phenomena of SMA strain-stress curves, and the dynamic model of SMA intravascular stent subjected to radial and axial stochastic excitations is established. The conditions of global stochastic stability of the system are determined, and the stationary probability density function of the system response is obtained. Finally, the stochastic Hopf bifurcation characteristics of the system are analyzed. Theoretical analysis and numerical simulation show that the system stability varies with bifurcation parameters, and stochastic Hopf bifurcation occurs in the process; there are two limit cycles in the stationary probability density of the system response in some cases, which means that there are two vibration amplitudes whose probability are both very high; jumping phenomena between the two vibration amplitudes appears with the change of conditions, which can cause stent fracture or loss. The results of this paper are helpful for application of SMA intravascular stent in biomedical engineering fields.

\section{Acknowledgment}

The authors gratefully acknowledge the support of the Natural Science Foundation of China (NSFC) through Grant Nos. 11272229 and 11302144, the Ph.D. Programs Foundation of the Ministry of Education of China through Grant No. 20120032120006, and the Tianjin Research Program of Application Foundation and Advanced Technology through Grant No. 13JCYBJC17900, 14JCQNJC05300, and 14JCQNJC05600.

\section{References}

[1] J. L. Longas, Design characteristics and mechanical properties of a new NiTi stent, Proceeding of the 2nd International Conference of Shape Memory and Superelastic Technolog 1 (1997), 567-570. 
[2] K. Kaoriuri, T. Koichi and Y. Zhong, Self-deployable origami stent grafts as a biomedical application of Ni-rich TiNi shape memory alloy foil, Materials Science and Engineering 1 (2006), 131-137.

[3] E. Verschuur, M. Homs and E. Steyerberg, A new esophageal stent design (Niti-S stent) for the prevention of migration: a prospective study in 42 patients, Gastrointestinal Endoscopy 1 (2006), 134-140.

[4] S. Puertolas, E. Bajador and J. Puertolas, Study of the behavior of a bell-shaped colonic self-expandable NiTi stent under peristaltic movements, BioMed Research International 4 (2013), 370582-6.

[5] F. Auricchio, M. Conti and S. Morganti, Shape memory alloy: from constitutive modeling to finite element analysis of stent deployment, Computer Modeling in Engineering and Sciences 3 (2010), 225-243.

[6] Z.W. Zhu, W.Y. Xie and J. Xu, Nonlinear dynamic characteristics of SMA simply supported beam in axial stochastic excitation, Journal of Vibroengineering 3 (2012), 1059-1063.

[7] E.J. Graesser and F.A. Cozzarelli, A proposed three-dimensional constitutive model for Shape memory alloys, Journal of Intelligent Material Systems and Structures 5 (1994), 78-89.

[8] Y. Ivshin and T.J. Pence, Thermomechanical model for a one variant shape memory material, Journal of Intelligent Material Systems and Structures 5 (1994), 455-473. 